\title{
Comparison of a manual walking platform and the CatWalk gait analysis system in a rat osteoarthritis model
}

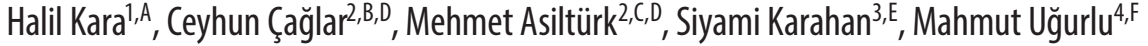 \\ ${ }^{1}$ Pharmacology Department, Ankara YıIdırım Beyazit University, Turkey \\ 2 Orthopaedics and Traumatology Department, Ankara City Hospital, Turkey \\ ${ }^{3}$ Veterinary Histology and Embryology Department, Kırıkkale University, Turkey \\ ${ }^{4}$ Orthopaedics and Traumatology Department, Ankara Yıldırım Beyazıt University, Turkey \\ A - research concept and design; $\mathrm{B}$ - collection and/or assembly of data; $\mathrm{C}$ - data analysis and interpretation; \\ $D$ - writing the article; $E$ - critical revision of the article; $F$ - final approval of the article
}

\section{Address for correspondence \\ Ceyhun Çağlar \\ E-mail: ceyhun.caglar@hotmail.com}

\section{Funding sources}

None declared

\section{Conflict of interest}

None declared

Received on March 10, 2021

Reviewed on May 2, 2021

Accepted on May 17, 2021

Published online on August 12, 2021

\section{Cite as}

Kara H, Çağlar C, Asiltürk M, Karahan S, Uğurlu M. Comparison of a manual walking platform and the CatWalk gait analysis system in a rat osteoarthritis model. Adv Clin Exp Med. 2021;30(9):949-956.

doi:10.17219/acem/137536

DOI

10.17219/acem/137536

\section{Copyright}

Copyright by Author(s)

This is an article distributed under the terms of the

Creative Commons Attribution 3.0 Unported (CC BY 3.0)

(https://creativecommons.org/licenses/by/3.0/)

\begin{abstract}
Background. Effects of osteoarthritis (OA) are observed in experimental animal models using different gait analysis systems.

Objectives. The aim of this study was to determine whether the Noldus CatWalk XT v. 10.9 gait analysis system (CatWalk) device can be used effectively in a chemically induced rat OA model and to reveal the strengths and weaknesses of the system compared to manual gait analysis.

Materials and methods. Ten Wistar rats were run on a manual walking platform as well as on the CatWalk and the basal values were recorded. For OA induction, monosodium iodoacetate (MIA) was injected into the left knee of all rats under anesthesia. After a period of 4 weeks for $\mathrm{OA}$ development, the rats were again run on both the manual and CatWalk gait platforms. For manual gait analysis, the stride length, paw print width and paw print length were measured on both knees. In addition to these parameters, the average run speed, run duration, maximum contact intensity, paw print area, mean stance, and swing speed were measured on the left knee (affected knee) using the CatWalk device.
\end{abstract}

Results. Significant differences were observed in the stride width ( $p=0.0272)$, left stride length $(p=0.0344)$, and left paw print length $(p=0.0233$ ) recorded before and after $\mathrm{OA}$ via the manual walking platform. For CatWalk, a significant difference was detected in the left knee's average run speed $(p=0.0010)$, maximum contact intensity $(p=0.0155)$, paw print length $(p=0.0058)$, paw print width $(p=0.0324)$, and swing speed $(p=0.0066)$ based on data obtained before and after 0 A.

Conclusions. The CatWalk gait analysis system is suitable for the evaluation of $0 \mathrm{~A}$ rat models and related interventions. It also provides additional parameters compared to the manual system and minimizes humanrelated variation.

Key words: CatWalk, osteoarthritis, rat model, gait analysis, monosodium iodoacetate 


\section{Background}

Animal models, especially those involving rodents, play an important role in drug development studies. For instance, molecules are used in preclinical studies involving animals to assess their effects on a specific disease to minimize the associated risks for humans before clinical studies. ${ }^{1}$ In this context, gait analysis in animal models that respond to a specific treatment and behavioral models are highly important. ${ }^{2}$

Osteoarthritis (OA) is the most frequently seen form of arthritis and its prevalence increases with age. ${ }^{3}$ Old age and obesity are particularly significant risk factors for OA. ${ }^{4,5}$ Although OA most frequently affects the knee joints, it can be seen in any joint in the body, including hip, waist and finger joints. ${ }^{6}$ The main symptoms include joint pain, joint stiffness and swelling. ${ }^{7}$ Currently, clinicians consider $\mathrm{OA}$ as a failure of the entire joint structure, such that OA not only affects articular cartilage but also affects the subchondral bone, ligaments, joint capsule, synovial membrane, and periarticular muscles. By another definition, OA starts as a result of the mechanical failure of the joint, during which the joint makes an effort to repair the defect area and fix the abnormal joint biomechanics. ${ }^{8}$ For OA treatment, pharmacological, non-pharmacological and surgical options are available. However, none of the available treatments provide an absolute solution for OA. Thus, there have been continuous efforts to reveal the complete pathobiology of OA and develop better treatment options. The response of animal models to OA treatments is measured using various kinds of in vivo techniques and through postmortem evaluations.

In previous studies, various chemicals have been used for the induction of OA in animals. ${ }^{9}$ In the present study, monosodium iodoacetate (MIA) is used. Intraarticular injection of MIA is one of the animal models of chemically induced OA. As a metabolic inhibitor, MIA causes disruption of glycolysis in cells by inhibiting the glyceraldehyde3 -phosphate dehydrogenase enzyme. ${ }^{10}$ Subsequently, this leads to an increase in oxidative stress in the environment that induces loss of chondrocytes, resulting in reduction of cartilage thickness and osteolysis. ${ }^{11-13}$ This mechanism of action governs the basis of MIA-induced OA. These changes bring about histological and morphological modifications in the joint cartilage similar to the prognosis of OA patients. ${ }^{14,15}$ Many studies have been conducted using MIA-induced OA rat models. ${ }^{16-19}$

There are several methods of rodent spatiotemporal gait analysis, and these systems have significant advantages compared to the main inkpad methods used earlier. Unfortunately, the system array and reporting of multiple parameters frequently make gait analysis more difficult. The CatWalk XT v. 10.9 gait analysis system (CatWalk; Noldus Company, Wageningen, the Netherlands) is a gait analysis device suitable for rodents, especially rats and mice. The data obtained by walking a rat on a platform are automatically saved.

\section{Objectives}

The purpose of this study is to evaluate the gait analysis patterns of rats before and after onset of OA induced by MIA. Using the values before induction of OA, the rat model was evaluated in terms of how the gait parameters changed after OA induction using the CatWalk device, which is one of the most powerful gait analysis systems available for rats and mice. In addition, data collected using the CatWalk software were compared to those obtained manually on graph paper. Evaluation of this rat model of OA will provide a basis for future OA studies involving gait analysis.

\section{Materials and methods}

\section{Animals}

Our research was approved by the Research Ethics Committee for Animal Experiments of Kırıkkale University, Turkey. The study was carried out with 10 male conventional Wistar rats weighing approx. $300 \pm 31.3 \mathrm{~g}$ and aged 12-24 weeks. The rats were kept in individual cages and fed ad libitum. A pellet diet was used for food and refined tap water was provided in an autoclavable Makrolon bottle. Sawdust was used as the bedding material and cleaned 4 times per week. The rat holding room was maintained at $23^{\circ} \mathrm{C}$ with $60 \%$ humidity and a $12 \mathrm{~h} / 12 \mathrm{~h}$ light/dark cycle. The air was completely replaced 15 times per hour. Prior to the gait procedures and injections, food was restricted for $24 \mathrm{~h}$ and water was restricted for $6 \mathrm{~h}$. Rats were regularly run on the CatWalk platform prior to the study and were thus adapted to the device. The animals were numbered by marking their tails.

\section{Knee osteoarthritis pain model}

Anesthesia was achieved by injection of $100 \mathrm{mg} / \mathrm{kg}$ intramuscular ketamine (Alfamine ${ }^{\circledR}$; Egevet, Izmir, Turkey; $100 \mathrm{mg} / \mathrm{mL}$ ) and $8 \mathrm{mg} / \mathrm{kg}$ intramuscular xylazine (Alfazyne $^{\circledR}$; Egevet; $2 \%$ ) through a 30 -gauge needle. Under general anesthesia, the left knees of the rats were shaved and $0.2 \mathrm{mg}$ of MIA (Sigma-Aldrich, St. Louis, USA) in $10 \mu \mathrm{L}$ of sterile saline was injected. The solution was injected through the patellar ligament using a 27-gauge needle with the leg flexed at a $90^{\circ}$ angle at the knee. After surgery, we waited 4 weeks for the formation of chemically induced OA.

\section{Gait analysis}

We selected 2 different systems for gait analysis. The $1^{\text {st }}$ gait analysis system was a manual walking platform. Analysis with a manual platform involves the movement of the animal along a single inkpad followed by the measurement 


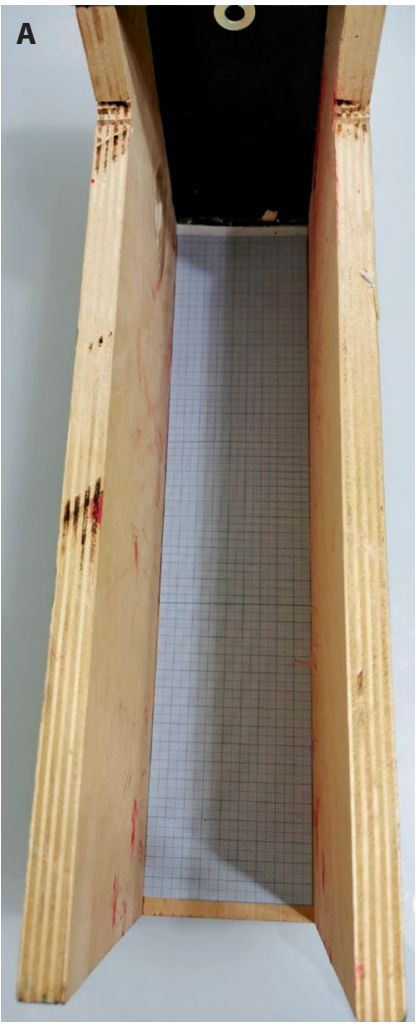

of the pattern of the ink prints on graph paper. Bait was placed at the end of the manual walking platform to encourage the rats. The rats walked in a dark room until they were accustomed to the manual walking platform (Fig. 1A,B). Once the rats were completely used to the platform and started walking, their hind paws were painted with ink. The rats were walked on graph paper and the values were recorded. The main data, such as stride length, stride width, paw print length, paw print width, and paw print area, are then measured from this paper. Variables such as the angle between the paws may also be assessed, but it should be kept in mind that these are not independent measurements and are derived from step length and width. ${ }^{20,21}$ Of note, the amount of ink is inconsistent between steps and trials and, therefore, the paw print areas are highly variable. Thus, modern high-speed videography has a higher probability of enabling robust analysis of spatial parameters.

The present study also used the CatWalk device for analysis using a machine learning-based approach. CatWalk is a gait analysis system for mice and rats that allows voluntary diagonal passage toward a target box over a glass surface in a darkened room, with the animals allowed to walk freely. ${ }^{22}$ A light from a lamp is aimed through the glass surface. When an animal's paw touches the glass surface, the light beams are reflected downward. The entire run is recorded with a video camera. When the rat walks, the paw prints are automatically captured, recorded and analyzed. ${ }^{23}$ This system has been explained in detail elsewhere. ${ }^{24}$ The animals walked on graph paper on the manual walking platform (Fig. 2) and the CatWalk device (Fig. 3A,B) in sequence before injection. All base

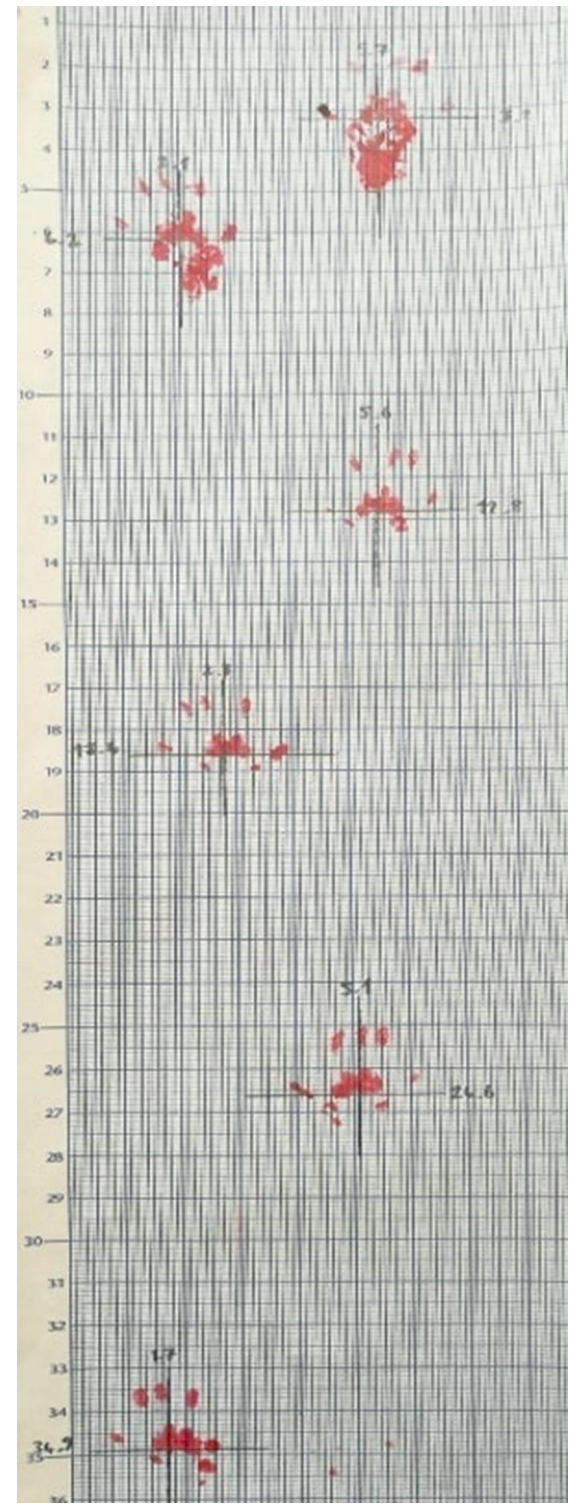

Fig. 2. Paw print paper used in manual testing 

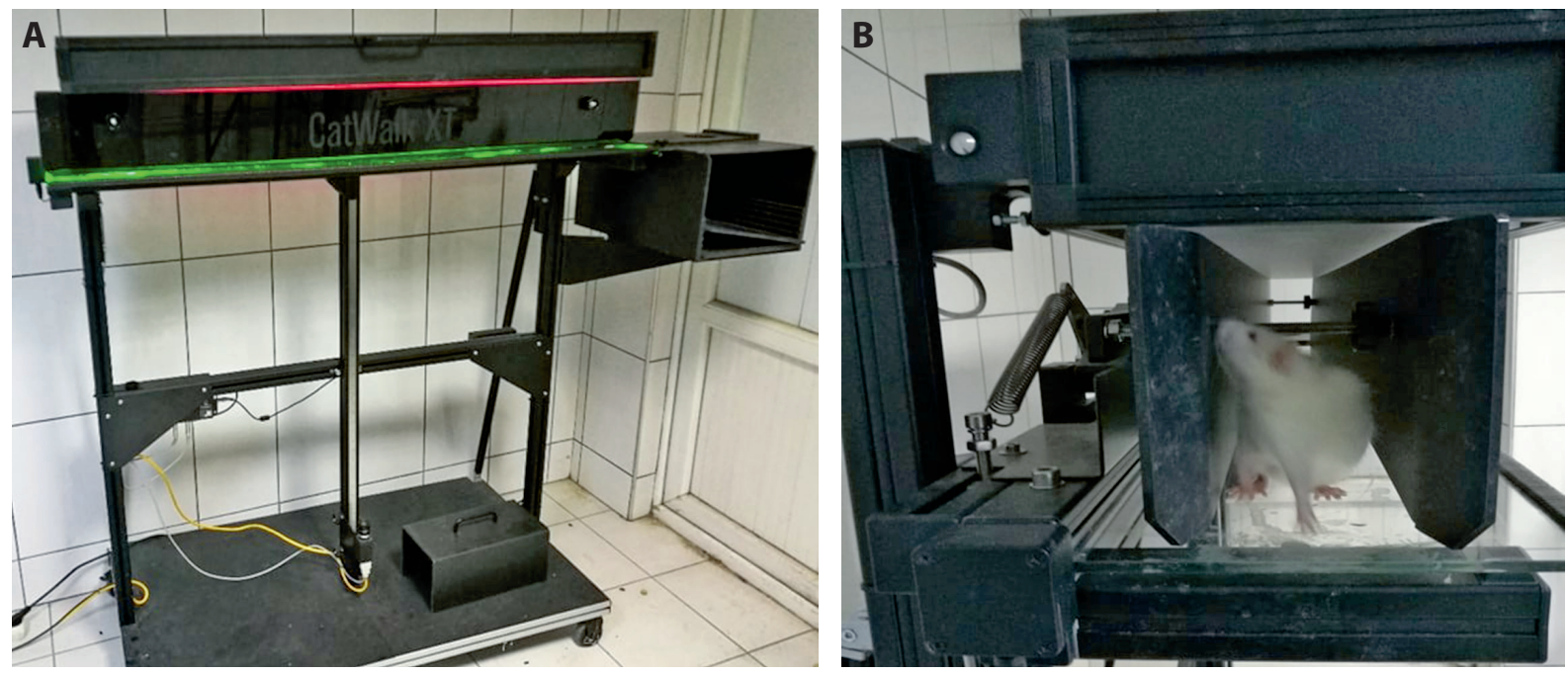

Fig. 3. A. The CatWalk hardware for gait analysis used for rats; B. A rat walking on the CatWalk platform

values were recorded for both platforms after this first walk. At this stage, the rats were administered MIA as mentioned above and 4 weeks were allowed for the formation of OA.

Four weeks after induction of OA, the animals were forced to walk again on the manual walking platform as well as the walking platform of the CatWalk device. Data collected both manually and via the CatWalk system before and after OA induction were categorized and analyzed. Data collected before and after OA induction were compared, and data collected manually and via CatWalk were analyzed for the presence of correlations.

With the analysis conducted using the CatWalk device, several parameters such as pre-treatment walking velocity, usage of the treated knee, comparisons with the other knees, run duration and average run speed, maximum contact area and intensity, stride length, paw print length, paw print width, paw print area, mean stance, and swing speed were analyzed by recording both baseline and OA values.

\section{Histological analysis}

All animals were euthanized by cervical dislocation after the second walk. The complete knee joint was collected. The joint samples were fixed in $10 \%$ phosphate-buffered formalin and then decalcified in 5\% formic acid. Decalcification was confirmed by radiography. Sections were then processed with routine histological techniques. The joints in paraffin blocks were cut to a thickness of $7 \mu \mathrm{m}$ and finally stained with toluidine blue (Fig. 4A,B) which enabled the researchers to evaluate loss of glycosaminoglycans in addition to morphology. The samples were then examined under a light microscope. The most severely affected regions of the joints were scored using the modified scoring system shown in Table 1. A total score for left knee joints was obtained and used in statistical analyses. Histological score data were expressed as median (minimum-maximum) scores.
Table 1. Rat osteoarthritis scoring system (0-25) 25

\begin{tabular}{|c|}
\hline Morphology (0-7) \\
\hline $\begin{array}{l}0=\text { normal } \\
1=\text { slight surface erosion or flaking of superficial zone } \\
2=\text { erosion no deeper than superficial zone } \\
3=\text { erosion into middle zone with or without fissuring } \\
4=\text { erosion into deep zone with or without fissuring } \\
5=\text { erosion into calcified zone } \\
6=\text { erosion into the subchondral bone (eburnation) } \\
7=\text { fibrous tissue on eburnated areas }\end{array}$ \\
\hline Tidemark (0-2) \\
\hline $\begin{array}{l}0=\text { normal } \\
1=\text { touched by blood vessels } \\
2=\text { crossed by blood vessels }\end{array}$ \\
\hline Doubling of tidemark \\
\hline $\begin{array}{l}0=\text { normal }(\text { a basophilic line }) \\
1=\text { doubled }\end{array}$ \\
\hline Glycosaminoglycan loss (0-5) \\
\hline $\begin{array}{l}0=\text { normal } \\
1=\text { increased in all layers of articular cartilage } \\
2=\text { significantly decreased or no deeper than superficial zone } \\
3=\text { significantly decreased or absent, no deeper than middle zone } \\
4=\text { significantly decreased or absent, no deeper than tidemark } \\
5=\text { no staining at all }\end{array}$ \\
\hline Chondrocyte morphology (0-5) \\
\hline $\begin{array}{l}0=\text { normal } \\
1=\text { enlarged cells close to the surface of articular cartilage } \\
2=\text { hypercellular with or without small clones } \\
3=\text { noticeable hypocellularity with or without clones } \\
4=\text { significant hypocellularity with or without clones } \\
5=\text { severe hypocellularity }\end{array}$ \\
\hline Osteophyte formation (0-2) \\
\hline $\begin{array}{l}0=\text { none } \\
1=\text { extensive mix tissue formation and remodeling at joint margin } \\
2 \text { = osteophyte }\end{array}$ \\
\hline Synovitis (0-4) \\
\hline $\begin{aligned} 0= & \text { normal }(1-\text { to } 3 \text {-cell-thick synovium and few mononuclear cells in } \\
& \text { subintima) } \\
1= & \text { slight increase in number of synoviocytes and mononuclear cells } \\
2= & \text { mononuclear cell infiltration and hyperemic blood vessels } \\
3= & \text { hyperplastic synovium } \\
4= & \text { extensive hyperplasia with pannus formation }\end{aligned}$ \\
\hline
\end{tabular}



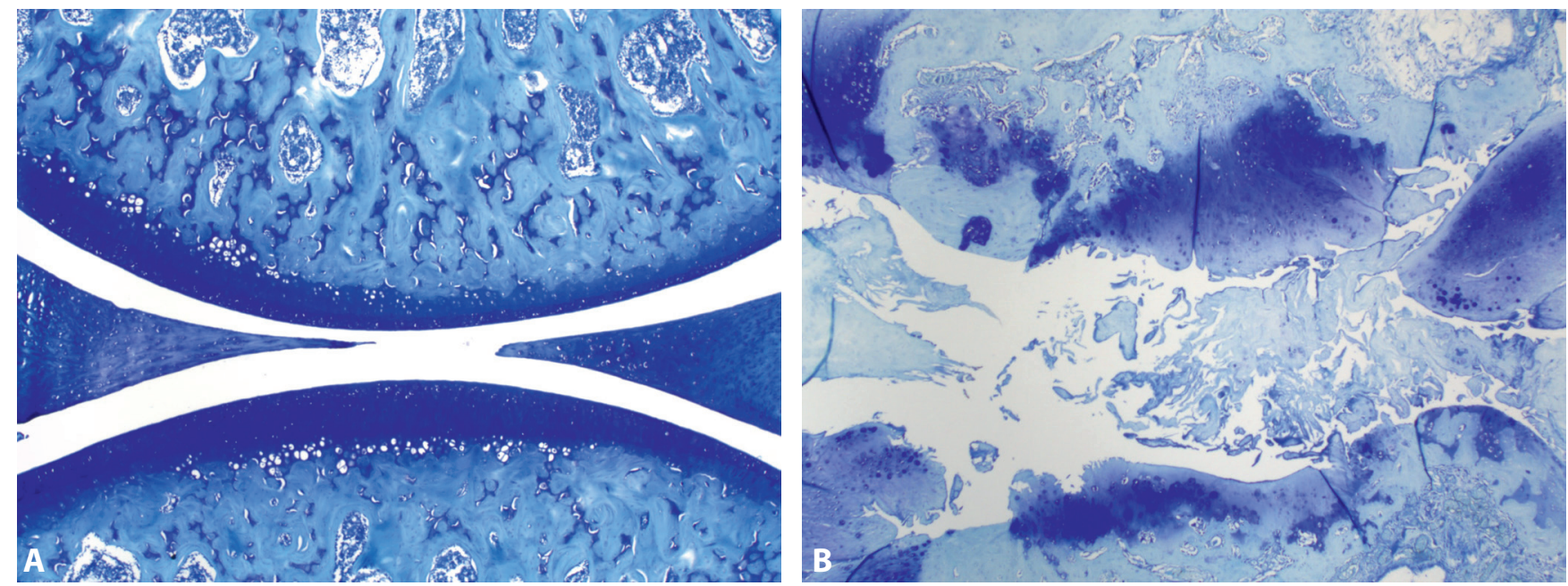

Fig. 4. A. Microscopic view of a cross-section of a toluidine-stained knee joint of a rat before the induction of osteoarthritis; B. Microscopic view of a crosssection of a toluidine-stained knee joint of a rat after the induction of osteoarthritis. Note the severe cartilage damage in the femorotibial joint

Table 2. Values of the rats' left (affected) and right (unaffected) knees measured from manual walking platform plotting paper

\begin{tabular}{|l|c|c|c|}
\multicolumn{1}{|c|}{ Parameter } & Base values, median (min-max) & After OA values, median (min-max) & $p$-value* \\
\hline Stride width $[\mathrm{cm}]$ & $3.35(3.00-4.10)$ & $4.20(3.90-4.70)$ & 0.0272 \\
\hline Right stride length $[\mathrm{cm}]$ & $11.10(9.70-12.30)$ & $12.85(11.20-14.10)$ & 0.3288 \\
\hline Left stride length $[\mathrm{cm}]$ & $11.90(10.20-14.0)$ & $10.35(9.30-12.90)$ & 0.0344 \\
\hline Right paw print width $[\mathrm{cm}]$ & $0.85(0.70-1.0)$ & $1.25(1.0-1.40)$ & 0.4267 \\
\hline Left paw print width [cm] & $1.05(0.90-1.20)$ & $0.90(0.70-0.90)$ & 0.1241 \\
\hline Right paw print length $[\mathrm{cm}]$ & $0.95(0.80-1.20)$ & $1.25(1.0-1.5)$ & 0.2230 \\
\hline Left paw print length $[\mathrm{cm}]$ & $1.30(1.30-1.40)$ & $1.10(1.0-1.10)$ & 0.0233 \\
\hline
\end{tabular}

* Wilcoxon rank-sum test; $\mathrm{n}$ (number of rats): 10; $\mathrm{df}$ (degrees of freedom): 9; $\mathrm{p}<0.05$ statistically significant; OA - osteoarthritis

\section{Statistical analyses}

Data are presented as median (minimum-maximum) values. The Wilcoxon rank-sum test, which is a non-parametric test, was used to assess whether or not there was a significant difference between both the base and after OA values of distance and histological OA scores between control and MIA-injected knees. The analyses were carried out using IBM SPSS Statistics for Windows v. 21 (IBM Corp., Armonk, USA). A value of $\mathrm{p}<0.05$ was accepted as significant in all statistical test.

\section{Results}

In histological sections, MIA-injected knees exhibited signs of OA. Surface erosion, fibrillation, clone formation, eburnation, tidemark invasion, and synovitis were common findings. The OA scoring system used for the rats in this study is shown in Table 1 . While the average OA score in the MIA-injected knee was 21.4 (18-25), the OA score in the control knee was $0.7(0-3)$. The OA scores in the MIA-injected knees were significantly higher compared to those in the control knees $(\mathrm{p}<0.05)$.
Seven parameters were evaluated with the manual walking platform. The calculated values are shown in Table 2 . After induction of OA, the) stride length of the left (affected) knee and the left paw print width and length values decreased; at the same time, the stride length of the right (unaffected) knee, right paw print width and length, and stride width increased. Among the data recorded from the manual walking platform, the values of stride width, left stride length and left paw print length were statistically significant.

The CatWalk software provided data for 9 different parameters. As the injections were administered to the left knee in all animals, values pertaining to the left hind limb were prioritized for analysis. The recorded values from the CatWalk device are shown in Table 3. After induction of $\mathrm{OA}$, the run duration and mean stance values of the left knee increased; at the same time, the average run speed, maximum contact intensity, paw print length, paw print width, paw print area, stride length, and swing speed of the same knee decreased. Among the data recorded with the CatWalk device, the average run speed, maximum contact intensity, paw print length, paw print width, and swing speed values were statistically significant. 
Table 3. Values of the left (affected) knee of rats obtained base and post OA parameters in CatWalk gait analysis system

\begin{tabular}{|l|c|c|c|}
\multicolumn{1}{|c|}{ Parameter } & Base values, median (min-max) & After OA values, median (min-max) & p-value* \\
\hline${ }^{1}$ Run duration $[\mathrm{s}]$ & $2.55(1.75-3.38)$ & $3.00(2.45-4.10)$ & 0.1077 \\
\hline${ }^{2}$ Run average speed $[\mathrm{cm} / \mathrm{s}]$ & $35.25(29.85-41.15)$ & $32.28(25.78-38.46)$ & 0.0010 \\
\hline${ }^{3}$ Mean stance $[\mathrm{s}]$ & $0.15(0.11-0.20)$ & $0.21(0.14-0.27)$ & 0.4054 \\
\hline${ }^{4}$ Maximal contact intensity [\%] & $97.00(92.50-100.00)$ & $75.00(68.50-81.00)$ & 0.0155 \\
\hline${ }^{5}$ Paw print length $[\mathrm{cm}]$ & $1.00(0.88-1.17)$ & $0.75(0.62-0.85)$ & 0.0058 \\
\hline${ }^{6}$ Paw print width $[\mathrm{cm}]$ & $0.90(0.82-0.95)$ & $0.70(0.60-0.75)$ & 0.0324 \\
\hline${ }^{7}$ Paw print area $\left[\mathrm{cm}{ }^{2}\right]$ & $0.40(0.35-0.48)$ & $0.32(0.25-0.40)$ & 0.3895 \\
\hline${ }^{8}$ Stride length $[\mathrm{cm}]$ & $9.80(8.92-10.47)$ & $8.95(8.20-9.45)$ & 0.3333 \\
\hline${ }^{9}$ Swing speed $[\mathrm{cm} / \mathrm{s}]$ & $148.40(119.10-176.20)$ & $95.30(72.40-102.70)$ & 0.0066 \\
\hline
\end{tabular}

Data are presented as median (min-max); ${ }^{*}$ Wilcoxon rank-sum test; $\mathrm{n}$ (number of rats): 10; $\mathrm{df}$ (degrees of freedom): 9; $\mathrm{p}<0.05$ statistically significant; OA -osteoarthritis. ${ }^{1}$ The time in which the rat crosses the walking platform. ${ }^{2}$ The speed of the rat on the walking platform. ${ }^{3}$ The contact time of the paw on the plate while walking. ${ }^{4}$ The degree of contact with the glass plate. ${ }^{5}$ The length that the paw occupied when it touched the walkway. ${ }^{6}$ The width that the paw occupied when it touched the walkway. ${ }^{7}$ The space that the paw occupied when it touched the walkway. ${ }^{8}$ The distance between steps of the same paw. ${ }^{9}$ The speed of a paw while it was in the air.

\section{Discussion}

There are many different systems that can be used for automatic gait analysis. ${ }^{26}$ These include DigiGait, TreadScan, the open-source Experimental Dynamic Gait Arena for Rodents, and CatWalk. In a study by Xu et al., the DigiGait and CatWalk gait analysis systems were compared and the advantages of each were reported. ${ }^{27}$ Five different automatic gait analysis systems were compared in a study by Jacobs et al., who mentioned that these devices play an important role in rodent OA models. ${ }^{28}$ In the present study, we compared manual platform data analysis and automated CatWalk gait data analysis.

The CatWalk gait analysis system allows simultaneous analysis of several factors by recording the steps of rats walking on a band, categorizing these steps and creating dozens of parameters. ${ }^{29}$ Data regarding all of the parameters, which are collected by the software, were statistically analyzed in this study. Upon the detection of significant differences, data were compared between groups. The CatWalk device can provide many more types of data, and the software is fast and easy to use. In this sense, it was observed that the CatWalk device has clear advantages over the manual platform method in terms of gait analysis. In addition to the parameters measured by classical methods, CatWalk can evaluate parameters that we think are important for OA within the walking analysis system. ${ }^{30}$

The MIA administration is an important method for chemical induction of OA. ${ }^{16}$ Other methods of chemical OA described in the literature include anterior cruciate ligament transection, destabilization of the medial meniscus and the use of collagenase. ${ }^{31}$ For example, Adães et al. used collagenase to create a rat model of $\mathrm{OA},{ }^{32}$ while Jacobs et al. used surgical medial meniscus and anterior cruciate ligament transection to create a similar OA model..$^{33}$ Most importantly, the MIA OA model progresses with a pathology similar to that of degenerative OA. ${ }^{13,34,35}$ Thus, we preferred the MIA model for OA induction. Signs of OA were observed in the left hind limbs of all rats following MIA administration in our study.

In the analysis, the first marked evidence was the presence of differences between data collected prior to and after OA induction. Such differences were obtained from both the CatWalk and the manual platform. Therefore, the parameters used in the CatWalk gait analysis system can be considered to detect gait changes in the MIAinduced rat OA model, a successful and well-established OA model documented in the literature. ${ }^{36}$

In the measurements after OA induction, it was observed that the rats deliberately avoided putting weight on their left hind limbs and possibly felt pain. ${ }^{37}$ The average run speed and swing speed decreased and, secondarily to this, the run duration and main stance increased. Moreover, the decrease in the length, width, and area of the paw prints and stride length after $\mathrm{OA}$ induction suggest that the rats stepped on the platform less widely to reduce the pressure on the foot of the affected limb. This hypothesis is also supported by decreases in the maximum contact density values. Whereas paw print measurements on the manual walking platform are performed manually with the help of a ruler, paw print measurements in the CatWalk system are automatically analyzed and provided by the software. Thus, the CatWalk system is more convenient and more objective than the manual walking platform. Gabriel et al. used the CatWalk software to assess acute inflammatory pain model rats and reported that they obtained objective and detailed data. ${ }^{38}$ In another study, rats with complete Freund's adjuvant-induced monoarthritis were analyzed using CatWalk software and clearer and non-biased results were also obtained. ${ }^{39}$ All of these results support that the parameters used by the CatWalk software are valuable for monitoring in vivo OA progression as well as responses to any intervention in $\mathrm{OA}$. 


\section{Limitations}

Our study has some limitations. First, the measurements from the manual walking platforms were obtained using a completely hand-held manner and may thus contain intra-observer and inter-observer measurement errors. One disadvantage of the CatWalk gait analysis system is that only rats and mice can be used. Also, rodents need a certain amount of time to adjust to the walking platform. Since the study was carried out in a dark room, the rodents behaved shyly in the environment and required a period of serious acclimatization.

\section{Conclusions}

In conclusion, the CatWalk gait analysis system is a useful, reliable and convenient method for monitoring gait changes in rat knee OA models. The CatWalk gait analysis system and its associated parameters, such as stride length, paw print width, paw print length, average run speed, run duration, maximum contact intensity, paw print area, mean stance, and swing speed, are appropriate for the evaluation of rat knee OA models.

\section{ORCID iDs}

Halil Kara (D) https://orcid.org/0000-0002-7557-7897

Ceyhun Çağlar (i) https://orcid.org/0000-0003-4286-7852

Mehmet Asiltürk (D) https://orcid.org/0000-0002-1069-8743

Siyami Karahan (iD https://orcid.org/0000-0002-2744-1717

Mahmut Uğurlu (D) https://orcid.org/0000-0001-9968-5393

\section{References}

1. Cardillo TM, Govindan SV, Sharkey RM, et al. Humanized anti Trop-2 IgG-SN-38 conjugate for effective treatment of diverse epithelial cancers: Preclinical studies in human cancer xenograft models and monkeys. Clin Cancer Res. 2011;17(10):3157-3169. doi:10.1158/10780432.CCR-10-2939

2. Berryman ER, Harris RL, Moalli M, et al. Digigait quantitation of gait dynamics in rat rheumatoid arthritis model. JMusculoskelet Neuronal Interact. 2009;9(2):89-98. PMID:19516084

3. Huang Y, Liu X, Xu X, et al. Intra-articular injections of platelet-rich plasma, hyaluronic acid or corticosteroids for knee osteoarthritis: A prospective randomized controlled study. Orthopade. 2019;48(3): 239-247. doi:10.1007/s00132-018-03659-5

4. Litwic A, Edwards MH, Dennison EM, et al. Epidemiology and burden of osteoarthritis. BrMed Bull. 2013;105:185-199. doi:10.1093/bmb/ Ids038

5. Allen KD, Golightly YM. State of the evidence. Curr Opin Rheumatol. 2015;27(3):276-283. doi:10.1097/BOR.0000000000000161

6. Rafanan BS, Valdecañas BF, Lim BP, et al. Consensus recommendations for managing osteoarthritic pain with topical NSAIDs in AsiaPacific. Pain Manag. 2018;8(2):115-128. doi:10.2217/pmt-2017-0047

7. Zhang Y, Jordan JM. Epidemiology of osteoarthritis. Clin Geriatr Med. 2010;26(3):355-369. doi:10.1016/j.cger.2010.03.001

8. Arnstein PM. Evolution of topical NSAIDs in the guidelines for treatment of osteoarthritis in elderly patients. Drugs Aging. 2012;29(7): 523-531. doi:10.2165/11631550-000000000-00000

9. Bendele AM. Animal models of osteoarthritis. J Musculoskelet Neuronal Interact. 2001;1(4):363-376. PMID:15758487

10. Guzman R, Evans M, Bove S, et al. Mono-iodoacetate-induced histologic changes in subchondral bone and articular cartilage of rat femorotibial joints: An animal model of osteoarthritis. Toxicol Pathol. 2003;31(6):619-624. doi:10.1080/01926230390241800
11. Pomonis JD, Boulet JM, Gottshall SL, et al. Development and pharmacological characterization of a rat model of osteoarthritis pain. Pain. 2005;114(3):339-346. doi:10.1016/j.pain.2004.11.008

12. Mannelli LDC, Bani D, Bencini A, et al. Therapeutic effects of the superoxide dismutase mimetic compound MnllMe2DO2A on experimental articular pain in rats. Mediators Inflamm. 2013;2013:905360. doi:10. $1155 / 2013 / 905360$

13. Kobayashi K, Imaizumi R, Sumichika H, et al. Sodium iodoacetateinduced experimental osteoarthritis and associated pain model in rats. J Vet Med Sci. 2003:65(11):1195-1199. doi:10.1292/jvms.65.1195

14. van der Kraan PM, Vitters EL, van de Putte LB, et al. Development of osteoarthritic lesions in mice by "metabolic" and "mechanical" alterations in the knee joints. Am J Pathol. 1989;135(6):1001-1014. PMID:2556924

15. Abdel-Rahman RF, Abd-Elsalam RM, Amer MS, et al. Manjarix attenuated pain and joint swelling in a rat model of monosodium iodoacetate-induced osteoarthritis. Food Funct. 2020;11(9):7960-7972. doi:10.1039/d0fo01297a

16. Miyagi $M$, Ishikawa $T$, Kamoda $H$, et al. Efficacy of nerve growth factor antibody in a knee osteoarthritis pain model in mice. BMC Musculoskelet Disord. 2017;18(1):428. doi:10.1186/s12891-017-1792-x

17. Ishikawa G, Koya Y, Tanaka H, et al. Long-term analgesic effect of a single dose of anti-NGF antibody on pain during motion without notable suppression of joint edema and lesion in a rat model of osteoarthritis. Osteoarthritis Cartilage. 2015;23(6):925-932. doi:10.1016/j. joca.2015.02.002

18. Lakes EH, Allen KD. Quadrupedal rodent gait compensations in a low dose monoiodoacetate model of osteoarthritis. Gait Posture. 2018;63: 73-79. doi:10.1016/j.gaitpost.2018.04.023

19. Sa $L$, Wei $X$, Huang $Q$, et al. Contribution of salidroside to the relieve of symptom and sign in the early acute stage of osteoarthritis in rat model. J Ethnopharmacol. 2020;259:112883. doi:10.1016/j.jep.2020.112883

20. de Medinaceli L, Freed WJ, Wyatt RJ. An index of the functional condition of rat sciatic nerve based on measurements made from walking tracks. Exp Neurol. 1982;77(3):634-643. doi:10.1016/0014-4886 (82)90234-5

21. Varejão AS, Cabrita AM, Geuna S, et al. Toe out angle: A functional index for the evaluation of sciatic nerve recovery in the rat model. Exp Neurol. 2003;183(2):695-699. doi:10.1016/s0014-4886(03)00208-5

22. Bernardes D, Oliveira ALR. Comprehensive catwalk gait analysis in a chronic model of multiple sclerosis subjected to treadmill exercise training. BMC Neurol. 2017;17(1):160. doi:10.1186/s12883-017-0941-z

23. Herold $S$, Kumar $P$, Jung $K$, et al. CatWalk gait analysis in a rat model of multiple sclerosis. BMC Neurosci. 2016;17(1):78. doi:10.1186/s12868016-0317-0

24. Hamers FP, Lankhorst AJ, van Laar TJV, et al. Automated quantitative gait analysis during overground locomotion in the rat: Its application to spinal cord contusion and transection injuries. J Neurotrauma. 2001;18(2):187-201. doi:10.1089/08977150150502613

25. Karahan S, Kincaid SA, Kammermann JR, et al. Evaluation of the rat stifle joint after transection of the cranial cruciate ligament and partial medial meniscectomy. Comp Med. 2001;51(6):504-512. PMID:11924812

26. Lakes EH, Allen KD. Gait analysis methods for rodent models of arthritic disorders: Reviews and recommendations. Osteoarthritis Cartilage. 2016;24(11):1837-1849. doi:10.1016/j.joca.2016.03.008

27. $X u$ Y, Tian NX, Bai QY, et al. Gait assessment of pain and analgesics: Comparison of the DigiGait ${ }^{\mathrm{TM}}$ and CatWalk ${ }^{\mathrm{TM}}$ gait imaging systems. Neurosci Bull. 2019;35(3):401-418. doi:10.1007/s12264-018-00331-y

28. Jacobs BY, Kloefkorn HE, Allen KD. Gait analysis methods for rodent models of osteoarthritis. Curr Pain Headache Rep. 2014;18(10):456. doi:10.1007/s11916-014-0456-x

29. Jacquez $B$, Choi H, Bird CW, et al. Characterization of motor function in mice developmentally exposed to ethanol using the Catwalk system: Comparison with the triple horizontal bar and rotarod tests. Behav Brain Res. 2021;396:112885. doi:10.1016/j.bbr.2020.112885

30. Chen H, Du J, Zhang Y, et al. Establishing a reliable gait evaluation method for rodent studies. J Neurosci Methods. 2017;283:92-100. doi:10.1016/j.jneumeth.2017.03.017

31. Gerwin N, Bendele AM, Glasson S, et al. The OARSI histopathology initiative: Recommendations for histological assessments of osteoarthritis in the rat. Osteoarthritis Cartilage. 2010;18(Suppl 3):24-34. doi:10.1016/j.joca.2010.05.030 
32. Adães S, Mendonça M, Santos TN, et al. Intra-articular injection of collagenase in the knee of rats as an alternative model to study nociception associated with osteoarthritis. Arthritis Res Ther. 2014;16(1):R10. doi:10.1186/ar4436

33. Jacobs BY, Dunnigan K, Pires-Fernandes M, et al. Unique spatiotemporal and dynamic gait compensations in the rat monoiodoacetate injection and medial meniscus transection models of knee osteoarthritis. Osteoarthritis Cartilage. 2017;25(5):750-758. doi:10.1016/j. joca.2016.12.012

34. Fonsi M, El Amrani Al, Gervais F, et al. Intra-articular hyaluronic acid and chondroitin sulfate: Pharmacokinetic investigation in osteoarthritic rat models. Curr Ther Clin Exp. 2019;92:100573. doi:10.1016/j. curtheres.2019.100573

35. Park MH, Jung JC, Hill S, et al. FlexPro MD ${ }^{\circledR}$, a combination of krill oil, astaxanthin and hyaluronic acid, reduces pain behavior and inhibits inflammatory response in monosodium iodoacetate-induced osteoarthritis in rats. Nutrients. 2020;12(4):956. doi:10.3390/nu12040956
36. Tschon M, Salamanna F, Martini L, et al. Boosting the intra-articular efficacy of low dose corticosteroid through a biopolymeric matrix: An in vivo model of osteoarthritis. Cells. 2020;9(7):1571. doi:10.3390/ cells9071571

37. Zhang W, Moskowitz RW, Nuki G, et al. OARSI recommendations for the management of hip and knee osteoarthritis. Part II: OARSI evidence-based, expert consensus guidelines. Osteoarthritis Cartilage. 2008;16(2):137-162. doi:10.1016/j.joca.2007.12.013

38. Gabriel AF, Marcus MA, Honig WM, et al. The CatWalk method: A detailed analysis of behavioral changes after acute inflammatory pain in the rat. $J$ Neurosci Methods. 2007;163(1):9-16. doi:10.1016/j. jneumeth.2007.02.003.

39. Parvathy SS, Masocha W. Gait analysis of C57BL/6 mice with complete Freund's adjuvant-induced arthritis using the CatWalk system. BMC Musculoskelet Disord. 2013;14:14. doi:10.1186/1471-2474-14-14. 